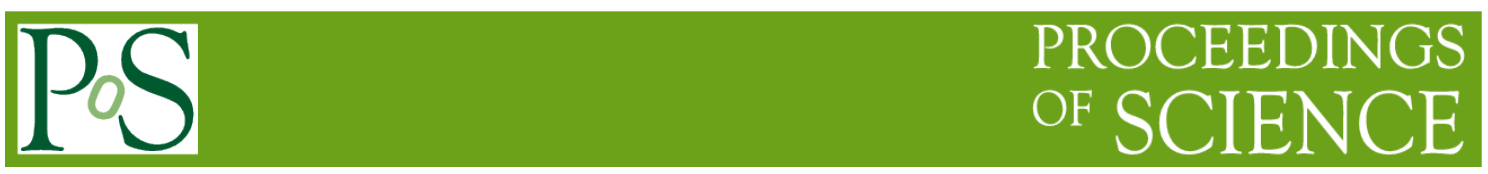

\title{
Hardened x-ray crystal spectrometer
}

\author{
C. Reverdin ${ }^{1}$, L. Lecherbourg, V. Dervieux, D. Gontier, B. Loupias \\ CEA, DAM, DIF \\ Bruyéres le Chatel F-91297, France \\ E-mail: charles.reverdin@cea.fr
}

\section{S. Baton}

LULI, Ecole polytechnique

Palaiseau, F-91128, France

In inertial confinement experiments, diagnostics will need to be protected against the effects of the harsh radiation environment. Either some heavy shielding must be included, or distance to the target must be increased suitably.

To effect the latter, polycapillary lenses can be used to focus divergent broadband x-ray radiation coming from a target into a collimated beam in order to transport x-rays a few meters up to the entrance of a spectrometer. An x-ray spectrometer has been built including a polycapillary lens, a curved crystal and a streaked camera.

This paper describes this diagnostic and will provide some preliminary results obtained in recent experiments at the LULI facility.

First EPs Conference on Plasma Diagnostics - $1^{\text {st }}$ ECPD

14-17 April 2015,

Villa Mondragone , Frascati (Rome) Italy

${ }^{1}$ Speaker 


\section{Introduction}

In inertial confinement experiments, diagnostics will need to be protected against the effects of the harsh radiation environment. Either some heavy shielding must be included, or distance from the target must be increased suitably. Since in $\mathrm{x}$-ray spectroscopic measurements signal level on the detector is often low, simply increasing distance is not an option.

For a few years now, broad band polycapillary lenses which transmit x-rays with a good efficiency in the $1-10 \mathrm{keV}$ energy range have been available commercially [1,2]. They are mainly used in x-ray fluorescence and spectrometry on small samples. They are used in astronomy to focus parallel beam broad band x-ray radiation and direct it to a diagnostic [3].

They could also be used in laser plasma diagnostics to focus divergent broadband $\mathrm{x}$-ray radiation coming from a target into a collimated beam. As an example reference [4] shows an $\mathrm{x}$ rays transport line composed of two polycapillary lens. They were used to separate the laserproduced x-ray source from the probe plasma sample by a distance of 0.9 meter, in order to greatly simplify the target holders.

These X-ray optics could be used to transport X-rays a few meters up to the entrance of a spectrometer. To prove the feasibility of this concept and explore the trade-off, an x-ray spectrometer has been built including a polycapillary lens, a curved crystal and a CCD camera or a streak camera.

This paper describes this diagnostic and will provide some preliminary results obtained in recent experiments at the LULI facility.

\section{Spectrometer design}

This spectrometer consists of two blocks:

1. Collimation block: a polycapillary lens, close to the target, transforms divergent $\mathrm{x}$-rays coming from the target to a collimated x-ray beam. The front of the polycapillary is protected against the target sputtering by a thin film.

2. Spectrometer: a filter, a crystal and a detector (IP, x-ray CCD camera or x-ray streak camera).

Two crystals were designed to record either an aluminium spectrum $(1500-2100 \mathrm{eV})$ or a titanium spectrum (4500-6300 eV) with a on-axis streak camera. The experiment was performed on the PICO2000 facility at LULI. The experimental conditions will be described in the results section. Figure 1 shows a schematic of the final design.

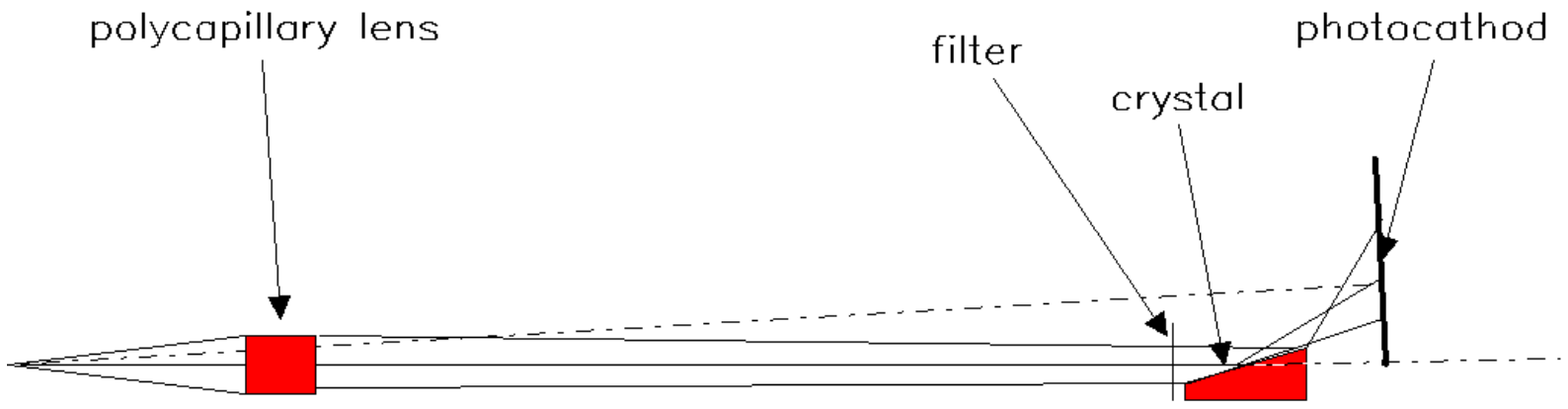

Fig. 1: Schematic of the design of this spectrometer 


\section{$2.1 \quad$ The polycapillary lens}

In future inertial confinement experiments, distance of the tip of a diagnostic must be bigger than $500 \mathrm{~mm}$ in order to be able to protect efficiently the first optic with consumable filters against the effects of the harsh radiation environment and the transport distance of $\mathrm{x}$-rays would be 5 to 10 meters. In smaller facilities such as LULI, conditions are not as harsh, experimental area are smaller and such large distances cannot easily be accommodated. So, to test the concept of this hardened spectrometer at LULI, smaller distances had to be chosen. Distance of the front of the polycapillary lens to the target has been chosen to be $200 \mathrm{~mm}$ and distance of the exit of the polycapillary lens to the crystal is $780 \mathrm{~mm}$.

Figure 2 shows a diagram of the collimating polycapillary lens. It has been produced by IFG [5], and it is composed of a bundle of $35 \mu \mathrm{m}$ hollow capillaries. The wall material is made of borosilicate glass. The transmission of this lens, $\mathrm{K}_{\mathrm{tr}}$, is high in the 1 to $10 \mathrm{keV}$ energy range and its value is $66 \%$ for cupper $\mathrm{K}_{\alpha}$ radiation.

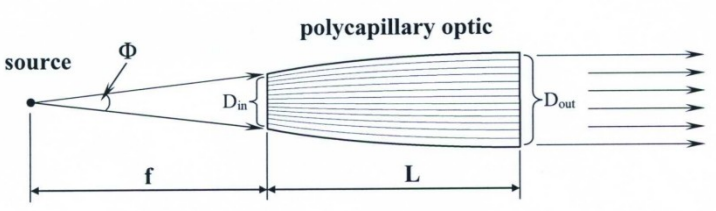

\begin{tabular}{|l|c|}
\hline f, $\mathbf{m m}$ & 200 \\
\hline $\mathrm{L}, \mathbf{m m}$ & 47.1 \\
\hline $\mathbf{D}_{\text {in }}, \mathbf{m m}$ & 16.5 \\
\hline$D_{\text {out }}, \mathbf{m m}$ & 18.8 \\
\hline $\mathrm{D}_{\text {cap }}, \boldsymbol{\mu m}$ & 35 \\
\hline$\Phi$, rad & 0.082 \\
\hline$\Delta \theta$, degr.(exit divergence - CuK $\alpha)$ & 0.25 \\
\hline $\mathrm{K} \tau \mathbf{r}, \%(\mathrm{CuK} \alpha)$ & 66 \\
\hline
\end{tabular}

Fig. 2: Characteristics of the polycapillary lens produced by IFG [5]

\section{$2.2 \quad$ The spectrometer}

The aim of the experiment at LULI was to record an aluminium spectrum (1500-2100 eV) and a titanium spectrum (4500-6300 eV) with a streak camera with a $15 \mathrm{~mm}$ length photocathode. To record these two ranges, a CsAP crystal was designed for aluminium and a PET crystal for titanium.

The incoming $\mathrm{x}$-ray beam from the polycapillary is non-perfectly collimated, and a flat crystal does not provide sufficient dispersion. A concave crystal should be used. In order to have a good spectral resolution, it is well known that a concave crystal of radius $\mathrm{R}_{\mathrm{C}}$ in the Johann geometry focuses monochromatic radiation on the Rowland circle of radius $R_{R}=R_{C} / 2$ [6].

When the detector is off the Rowland circle, the height of the monochromatic spot, $\mathrm{h}_{\mathrm{m}}$, is:

$$
h_{m}=h_{c} \frac{d_{2}-R_{C} \sin \left(\theta_{B}\right)}{d_{1}-R_{C} \sin \left(\theta_{B}\right)}
$$

where $\mathrm{h}_{\mathrm{c}}$ is the height of the $\mathrm{x}$-ray source $(\sim 20 \mathrm{~mm}), \mathrm{d}_{1}$ is the distance from the $\mathrm{x}$-ray source to a point on the crystal $(\sim 800 \mathrm{~mm}), \mathrm{d}_{2}$ is the distance from this point to the detector $(\sim 135 \mathrm{~mm})$, $\theta_{B}$ is the Bragg angle. The local Rowland circle of radius $R_{R}$ is tangent to the crystal at this point. Figure 3a shows a scheme of this geometry.

If the distance from the Rowland circle to the detector is $d_{2}-R_{c} \sin \left(\theta_{B}\right)=10 \mathrm{~mm}$, the height of the spot is $h_{m}=0.25 \mathrm{~mm}$. A large spectral range needs to be recorded with a small detector length $(15 \mathrm{~mm})$ and it was only possible to put the detector on axis. So the spectral range related to a distance from the Rowland circle to the detector smaller than $10 \mathrm{~mm}$ would be quite small and outside this range the spectral resolution would be quite poor. 
In order to improve this rather poor spectral resolution, the local curvature $\mathrm{R}$ has been chosen to have the local Rowland circle intersecting the detector at the Bragg angle $\theta_{\mathrm{B}}$ for each $\mathrm{x}$-ray energy. This allows all $\mathrm{x}$-rays coming from the polycapillary lens to be focused on the detector. The shape of the crystal has been deduced progressively, starting from one end of the crystal. The final shape is approximated by a $5^{\text {th }}$ order polynomial curve:

$$
y=0.20 x+6.7 \times 10^{-4} x^{2}+3.3 \times 10^{-6} x^{3}+5.0 \times 10^{-12} x^{4}+1.9 \times 10^{-10} x^{5}
$$
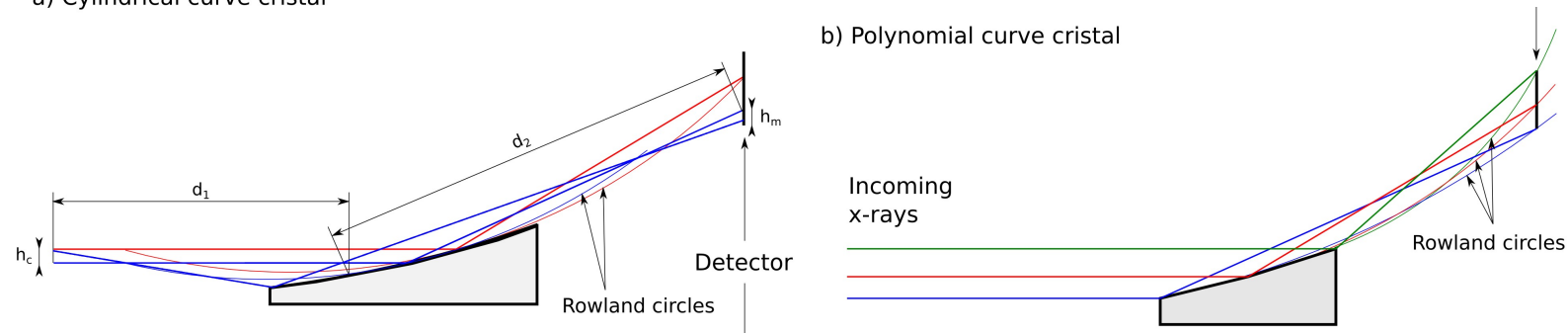

Fig. 3: Focusing of monochromatic radiation on the Rowland Circle for a) spherically bent crystal or b) polynomial bent crystal.

Figure $3 \mathrm{~b}$ presents a ray trace of the spectrometer with the Rowland circles in the middle of the crystal and at both ends. The main geometric characteristic of the spectrometer are in table 1.

\begin{tabular}{|l|c|c|c|}
\hline & high energy end & middle & low energy end \\
\hline $\mathbf{( X , Y},)_{\text {crystal }}[\mathbf{m m}]$ & $(-35,-9.6)$ & $(0,-1)$ & $(35,10.1)$ \\
\hline $\mathbf{R}_{\text {crystal }}[\mathbf{m m}]$ & 784 & 505 & 301 \\
\hline $\mathbf{( X , \mathbf { Y }})_{\text {detector }}[\mathbf{m m}]$ & $(115,57.8)$ & $(115,67.4)$ & $(115,81)$ \\
\hline $\mathbf{E}_{\text {csAP }}[\mathbf{e V}]$ & $2300 \mathrm{eV}$ & $1815 \mathrm{eV}$ & $1360 \mathrm{eV}$ \\
\hline $\mathbf{E}_{\text {PET }}[\mathbf{e V}]$ & $6775 \mathrm{eV}$ & $5344 \mathrm{eV}$ & $4000 \mathrm{eV}$ \\
\hline
\end{tabular}

Table 1: Geometric characteristics of the spectrometer

The crystals were produced by Saint Gobain Crystals [7]. The dispersion curves on the detector are displayed in figure 4.
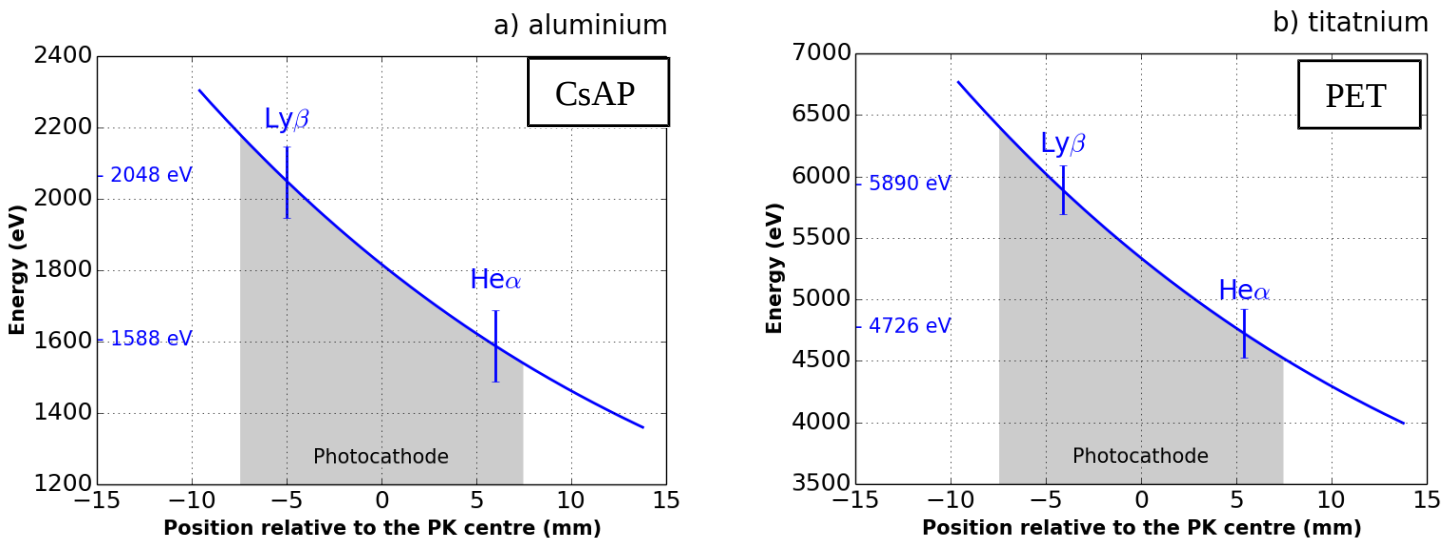

Fig. 4: Dispersion of the 2 crystals: a) CsAP crystal for aluminium range, b) PET crystal for titanium range. The grey shade shows the size of the photocathode.

\section{Experimental results}


Because the number of shots on LULI experiments is limited, an experiment to pre-align the lens and to check its divergence was performed on a small laser facility, EQUINOX [8]. A copper target has been irradiated with the EQUINOX laser, producing about $1 \mathrm{keV}$ radiation. The polycapillary lens has been put at the nominal distance from the target and orientation was optimized in order to have a high luminosity circular x-ray image on the CCD detector. The final orientation was referenced to be used as is on the LULI experiment.

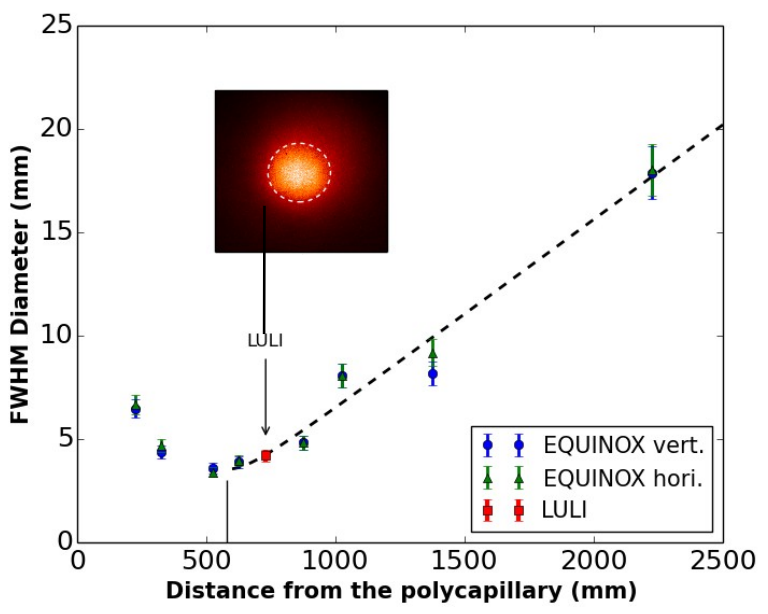

Fig. 5: Radius of the spot versus distance from the polycapillary lens. The blue and green markets are the data obtained at EQUINOX, and the red square is the result obtained during the LULI experiment.

During this experiment, we also recorded the size of the spot versus distance from polycapillary lens (figure 5). Images have been recorded with different distances from the exit of the lens to the detector. An unanticipated focusing about $580 \mathrm{~mm}$ from the exit of this lens was found. This focusing is due to a manufacturing issue. The FWHM at focus was measured to $3.5 \mathrm{~mm}$. The inset presents the raw image of the spot in front of the crystal's location during the LULI experiment, $750 \mathrm{~mm}$ from the pollycapillary lens.

For the LULI experiment, the x-ray beam was focused about $200 \mathrm{~mm}$ from the crystal and the FWHM of the x-rays was measured at $4.2 \mathrm{~mm}, 5 \mathrm{~cm}$ from the front of the crystal in agreement with EQUINOX measurements (red square in figure 5). So the crystal was not homogeneously illuminated.

Aluminium foils and titanium foils were irradiated with $5 \mathrm{~J}$ at $2 \omega$ in 1 ps. Recorded spectra are shown in figure 6. FWHM of $\mathrm{Ly}_{\alpha}$ lines of aluminium has been found to be $8 \mathrm{eV}$. The main effect of this unforeseen focusing is a shorter energy range and a hgher sensitivity in the middle of this energy range than at its edges, making measurement of ratio of lines unreliable.
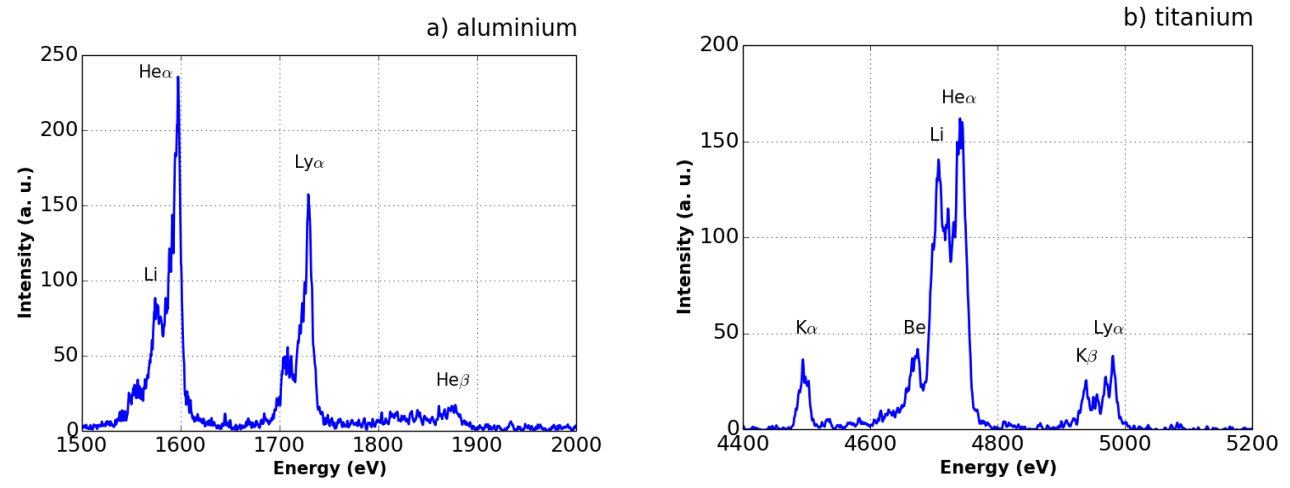

Fig. 6: Time-integrated spectra of a) aluminium and b) titanium. 
Time-resolved measurements were also performed. An example is shown in figure 7: a) the raw image was corrected to take into account difference of length between rays at low energy and rays at high energy, b) several line-out at three different times. The time resolution of such camera is estimated about 1 ps. Note that the dispersion is different than previously: the spectrometer was set here to record the four aluminium lines: $\operatorname{He} \alpha, \operatorname{Ly} \alpha, \operatorname{He} \beta$, and $\mathrm{Ly} \beta$.

a) raw data

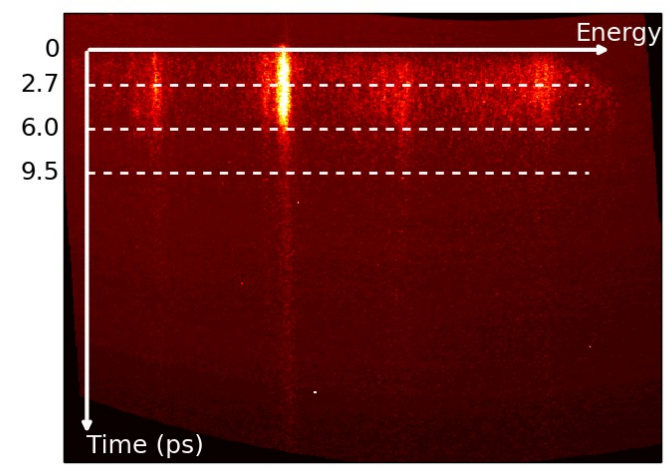

b) lines out

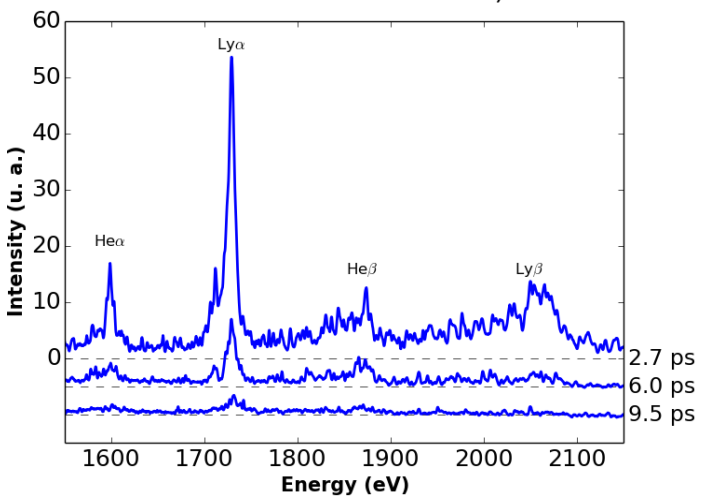

Fig. 7: Time-resolved spectrum of aluminium plasma: a) raw data and b) 3 different lines out.

\section{Conclusion}

We have shown experimentally that it is possible to couple a polycapillary lens with an $\mathrm{x}$-ray spectrometer at a distance of $1 \mathrm{~m}$ from the target. Although the polycapillary lens we bought was refocusing and so the intensity at the entrance of the spectrometer block was not homogeneous, this set-up allows to record a relatively wide spectral range $(>500 \mathrm{eV})$ on a streak camera slit $(<15 \mathrm{~mm})$. Such design could be updated to even increase the distance from the target in case of hardened spectrometer for very large facilities such as NIF or LMJ.

\section{Acknowledgements}

This work was supported by the Commissariat à l'Energie Atomique et aux Energies Alternatives (France). The authors would like to acknowledge the invaluable support of F. Serres from Ecole Polytechnique and of all the LULI facility operations staff and the technical team.

\section{References}

[1] M.A. Khumakov, Capillary optics and their use in x-ray analysis, X-ray spectroscopy Vol 29 343348 (2000)

[2] C.A. MacDonald, Applications and measurements of polycapillary lenses, Journal of x-ray science and technology 6 32-47 (1996)

[3] C.H. Russel and al., Polycapillary x-ray optics for x-ray astronomy, Advances in x-ray analysis, Vol 43, 480-486 (2000)

[4] F. Dorchies, N. Fedorov, and L. Lecherbourg, Experimental station for femtosecond-laser-based time-resolved $x$-ray absorption near-edge spectroscopy, Submitted to Rev. Sc. Instrum.

[5] Institute for Scientific Instruments GMBH, Rudower Chaussee 29/31, 12489 Berlin, Germany

[6] H.H. Johann, Die Erzeugung lichtstärker Röntgenspektrum mit Hilfe von Konkavkristalle, Z. Phys. 69,185 (1931)

[7] Saint Gobain Crystals, 77140 Saint Pierre les Nemours, France 
[8] C. Reverdin, M. Paurisse, T. Caillaud, P. Combis, A. Duval, D. Gontier, D. Husson, C. Rubbelynck, C. Züber, $X$-ray diagnostic calibration with the tabletop laser facility EQUINOX, Review of Scientific Instruments 79, 10E932 (2008) 